Conference Proceedings Paper - 1st International Electronic Conference on Molecular Science: Cell Signaling, Survival and Growth

\title{
Co-Presence of Mesenchymal Stem Cells In An In Vivo Engineered Liver Pocket-Scaffold Increases Neo-Angiogenesis And Albumin Production
}

\section{Amedo Carraro ${ }^{1}$, Maurizio Buggio ${ }^{2}$, Chiara Gardin ${ }^{3, *}$, Umberto Tedeschi ${ }^{1}$, Letizia Ferroni ${ }^{3}$ and Barbara Zavan ${ }^{3, *}$}

1 Department of General Surgery and Odontoiatrics, Liver Transplant Unit, University Hospital of Verona, P.le Aristide Stefani 1, 37126 Verona, Italy; E-Mails:

amedeo.carraro@ospedaleuniverona.it (A.C.); umberto.tedeschi@ospedaleuniverona.it (U.T.)

2 Nanomedicine Lab, Institute of Inflammation and Repair, Faculty of Medical \& Human Sciences, University of Manchester, Manchester M13 9PT, United Kingdom; E-Mail: maurizio.buggio@gmail.com (M.G.)

3 Department of Biomedical Sciences, University of Padova, Viale Giuseppe Colombo, 3, 35131 Padova, Italy; E-Mails: chiara.gardin@unipd.it (C.G.); letizia.ferroni@unipd.it (L.F.); barbara.zavan@unipd.it (B.Z.)

* Author to whom correspondence should be addressed; E-Mail: barbara.zavan@unipd.it (B.Z.); chiara.gardin@unipd.it (C.G.); Tel.: +39-49-827-6096; Fax: +39-49-827-6079.

Received: 23 July 2015 / Published: 13 October 2015

\begin{abstract}
The construction of a three-dimensional (3D) liver tissue is limited by many factors; one of them is the lack of vascularization inside the tissue-engineered construct. An engineered liver pocket-scaffold able to increase neo-angiogenesis in vivo could be a solution to overcome these limitations. In this work, a hyaluronan (HA)-based scaffold enriched with human Mesenchymal Stem Cells (hMSCs) and rat hepatocytes was preconditioned in a bioreactor system, then implanted into the liver of rats. Angiogenesis and hepatocytes metabolic functions were monitored. The formation of de novo vascular network within the HA-based scaffold as well as an improvement in albumin production by the implanted hepatocytes were detected. The presence of hMSCs in the HA-scaffold increased the concentration of growth factors promoting angiogenesis inside the graft. This event ensured a high blood vessel density coupled with a support to metabolic functions of hepatocytes. All together, these results highlight the important role played by stem cells in liver tissue-engineered engraftment.
\end{abstract}


Keywords: mesenchymal stem cell; hepatocyte; angiogenesis; hyaluronan-based scaffold; engineered liver tissue

\section{Introduction}

Liver transplantation still represents the only effective treatment for patients with liver failure. Unfortunately, the increasing demand for organs is so great that many patients die while awaiting transplantation [1]. In order to deal with this problem, many researchers have attempted to develop different extracorporeal bioartificial liver systems to provide a temporary support for exhausted liver; these are cell-based life support devices intended to enhance hepatic detoxification and protein synthesis functions in order to compensate for a whole liver [2-5]. However, such supports are limited and can only temporarily replace a subset of essential hepatic functions, suggesting the necessity to recapitulate all liver function by alternative approaches. De novo fabrication of three-dimensional (3D) liver tissue represents a potential strategy to cope with this problem and the shortage of donor organs; the existing results in tissue engineering emphasize the promising strategy to create in the future organs for transplantation. However, liver has a complex and defined organization in which hepatocytes and non-parenchymal cells are precisely arranged, and vascular and biliary networks are interdigitated in a highly aligned micro-architecture. In front of these aspects and the limitation related to the marked specialization of these cells, liver reconstruction is still limited by many factors; first of all the lack to recapitulate an adequate vasculature, which is able to maintain viable and functional cells outside the natural liver environment [6,7]. In the light of the fundamental role of environmental factors, translational research has directed many efforts to study the extracellular matrix (ECM) and remake its potential influences on cell survival and function [8,9]. A suitable perfusion of metabolically active tissue requires intimate localization of cells to a dense vasculature in a highly organized manner [10,11]. In light of these considerations, we have considered recent advances in the field of stem cell and nanotechnology research in order to produce a vascularized and functional tissue-engineered liver graft [12-16]. Advanced tissue engineering strategies were applied through the pre-conditioning of cell-seeded 3D scaffolds in a bioreactor system, followed by their implantation in an animal model. In particular, a hyaluronan (HA)-based scaffold was used as biodegradable platform to study the behavior of human Mesenchymal Stem Cells (hMSCs) in co-culture with hepatocytes.

\section{Results and Discussion}

\subsection{D-Cultures Preparation}

Our approach was based on the use of a bioreactor system to develop primarily 3D co-cultures of MSCs and hepatocytes onto HA-based tubes. HYAFF-11 $1^{\mathrm{TM}}$, a derivative of hyaluronic acid, was used as scaffold. This scaffold has previously found many applications in the biomedical field due to its low antigenicity, suitable biodegradability, and good mechanical and hemostatic properties [17]. Furthermore, this HA-based scaffold presents a great similarity with the structure of native ECM and is 
almost completely biodegradable ensuring the formation of neo-tissue with collagen and elastic fibers completely organized [18-20].

In particular, we have isolated rat hepatocytes and hMSCs from adipose tissue, then amplified them up to passage 3. After harvesting by trypsin treatment, the cells were seeded onto HA-based scaffold tubes in order to prepare three different combinations of 3D-cultures: mono-culture of rat hepatocytes, mono-culture of hMSCs, and co-culture of rat hepatocytes and hMSCs (ratio 1:1). All cell-seeded scaffolds were maintained in the bioreactor system at temperature and carbon dioxide controlled up to 4 days. Half of the cell-seeded scaffolds were maintained in the bioreactor for other 7 days, whereas the other half was implanted in rat models for other 7 days. The 3D-cultures maintained in vitro up to 11 days were analyzed through MTT and albumin ELISA assays at 4 and 7 days from cells seeding. On the contrary, the 3D-cultures implanted in rat models were analyzed by immunofluorescence (IF) and real-time PCR to detect albumin and vascular markers at 7 days from implantation.

\subsection{In Vitro Experiments}

The cell proliferation rate of the three 3D-cultures was quantified by means of the MTT assay (Figure 1a). A satisfactory cellular survival was demonstrated in all culture conditions at 4 days, confirming the efficacy of the described system. More detailed analyses evidenced that there was no detectable increase in MTT values over time in the mono-culture of rat hepatocytes. A significant increase over time in cell proliferation was observable in scaffolds containing hMSC alone, whereas a slight increase was detected in the co-culture of rat hepatocytes and hMSCs.

The cellular functionality of 3D-cultures was evaluated by quantification of the secreted albumin in culture media through an ELISA assay (Figure 1b). The albumin secretion was observed only in monoculture of rat hepatocytes and in the co-culture of rat hepatocytes and hMSCs. In particular, the albumin production by hepatocytes resulted strongly increased when hepatocytes were co-cultured with hMSCs. This preliminary in vitro result suggests that hMSCs could have the ability to support and influence the albumin synthesis of hepatocytes.

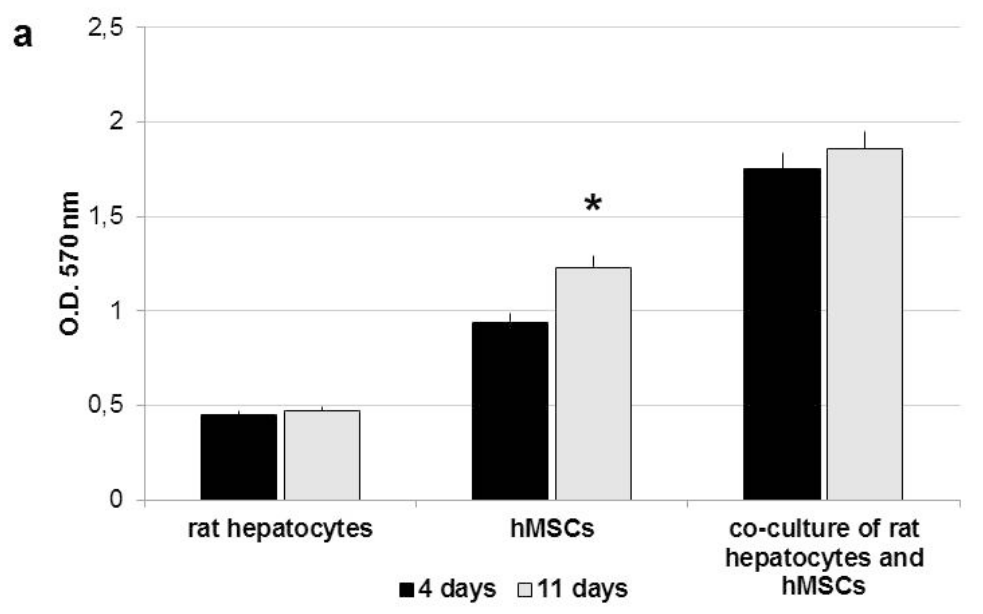

Figure 1. Cont. 


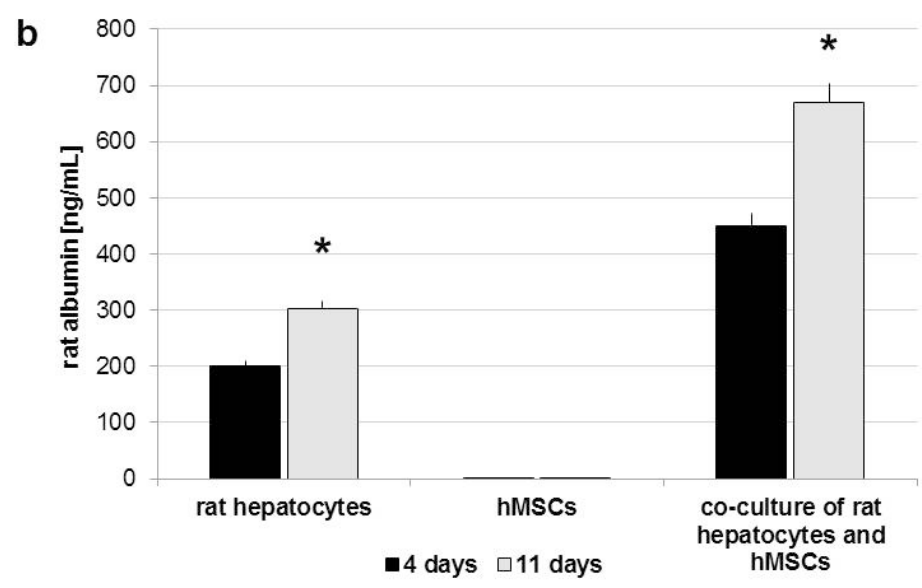

Figure 1. (a) MTT assay; (b) albumin ELISA assay on 3D-cultures of rat hepatocytes, hMSCs, and rat hepatocytes with hMSCs (ratio 1:1) after 4 and 11 days of culture in the bioreactor system.

\subsection{In Vivo Experiments}

After 4 days of pre-conditioning in the bioreactor system, the three 3D-cultures were implanted in rat models. The pocket-scaffolds were explanted from animals after 7 days, then molecular and morphological analyses were performed.

The rat albumin production was detected by means of real-time PCR and IF staining (Figure 2). The gene expression profile revealed albumin mRNA in pocket-scaffolds derived from 3D mono-culture of rat hepatocytes and 3D co-culture of rat hepatocytes and hMSCs (Figure 2a). This finding confirmed that high level of albumin is reached only when hMSCs are present in the 3D scaffold. IF staining on pocket-scaffold enriched with rat hepatocytes and hMSCs also confirmed the presence of rat albumin (green) around the HA fibers (black) of the scaffold (Figure 2b).
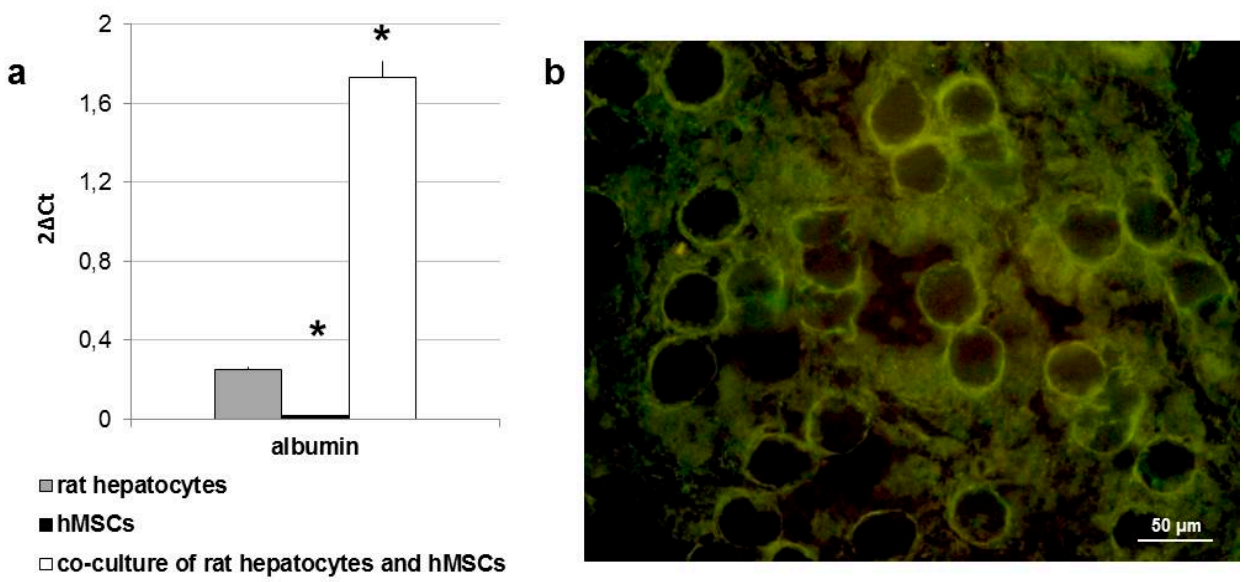

Figure 2. (a) Rat albumin mRNA in pocket-scaffolds derived from 3D mono-culture of rat hepatocytes, 3D mono-culture of hMSCs, or 3D co-culture of rat hepatocytes and hMSCs at 7 days from implantation in a rat model; (b) IF staining of rat albumin (green) in pocket-scaffold (black) enriched with rat hepatocytes and hMSCs at 7 days from implantation in a rat model. 
The presence of blood vessels in the pocket-scaffolds were investigated by mRNAs quantification and IF staining of endothelial markers (Figure 3).

Gene expression analysis reported in Figure 3 a shows the mRNA levels of the endothelial markers CD31 (alias PECAM1, platelet endothelial cell adhesion molecule), vascular endothelial growth factor A (VEGFA), vascular endothelial growth factor receptor 1 (VEGFR1 alias FLT1), vascular endothelial growth factor receptor 2 (VEGFR2 alias KDR), and von Willebrand factor (VWF). These endothelial markers were mainly detected in pocket-scaffolds consisting of hMSCs alone or in association with hepatocytes.
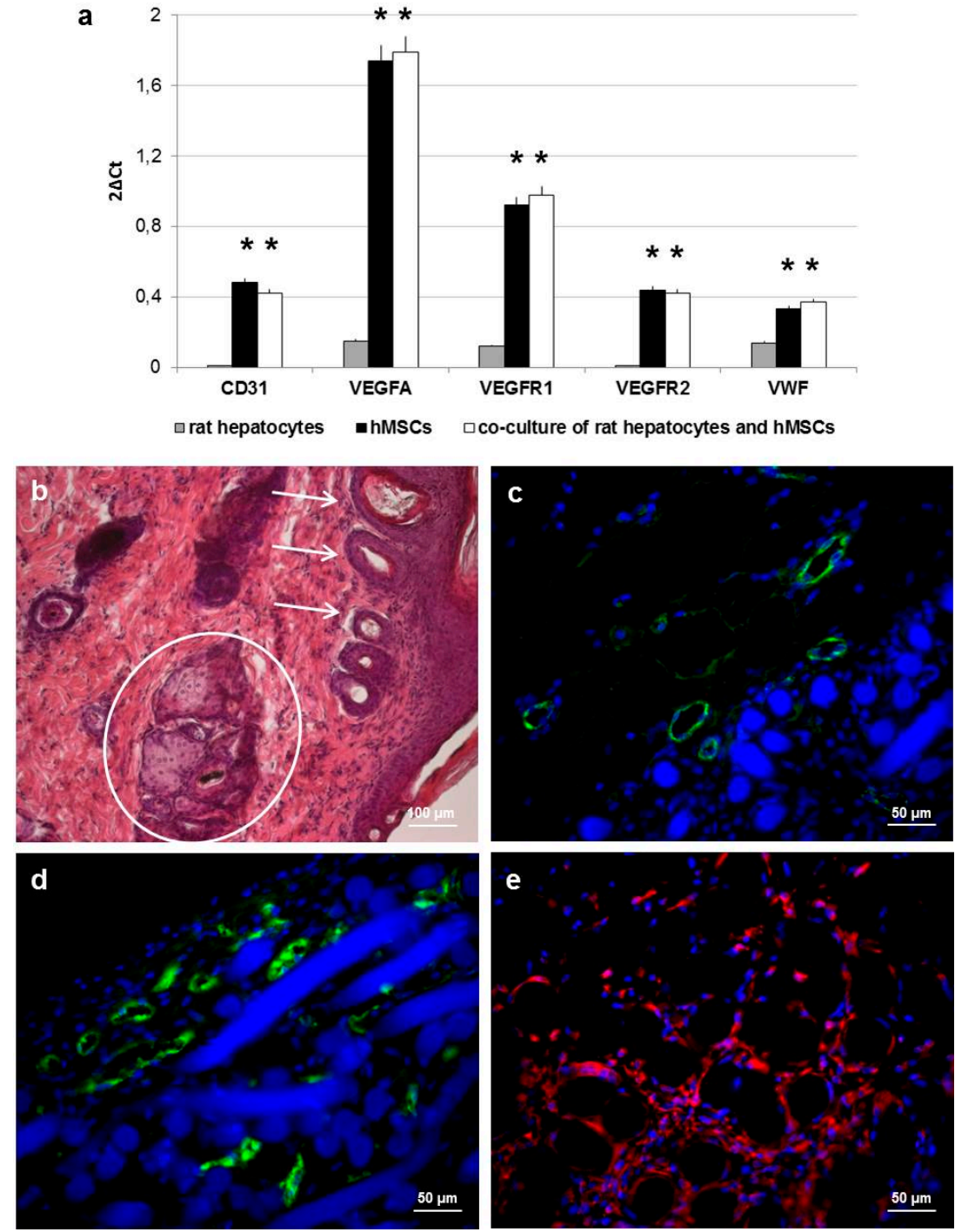

Figure 3. (a) Gene expression profiles of endothelial markers in pocket-scaffolds derived from 3D mono-culture of rat hepatocytes, 3D mono-culture of hMSCs, and 3D co-culture of rat hepatocytes and hMSCs at 7 days from implantation in a rat model; (b) H/E staining of pocket-scaffold enriched with rat hepatocytes and hMSCs showing rat hepatocytes (within the white circle) and several blood vessels (white arrows); IF staining of pocket-scaffold enriched with rat hepatocytes and hMSCs displaying (c) VWF (green) in the edge of the pocket-scaffold; (d) VWF (green) within the pocket-scaffold; (e) Vimentin (red) in the pocket-scaffold. Nuclei (blue) stained with Hoechst. 
The hematoxylin and eosin $(\mathrm{H} / \mathrm{E})$ staining of the pocket-scaffolds enriched with rat hepatocytes and hMSCs is reported in Figure 3b. The H/E staining showed that the rat hepatocytes (white circle) were clearly organized into clusters inside the HA fibers of the pocket-scaffold. Hepatocytes retained their characteristic phenotype with hexagonal shape often associated with double nuclei. A vascular network (white arrows) was revealed into the scaffold matrix up to $600 \mu \mathrm{m}$ apart from the edge. The earliest signs of angiogenesis were confirmed by the IF staining of VWF (green) that showed an high vessel density area in the edge of pocket-scaffold (Figure 3c), and vessels within the scaffold (Figure 3d). On the other hand, the presence of hMSCs was confirmed by IF staining of Vimentin (red in Figure 3e).

The morphological analyses demonstrated that in pocket-scaffolds consisting of rat hepatocytes and hMSCs the formation of new vessels occurred in the edge of the scaffold; furthermore, a significant migration of neo-developed vessels extended centripetally from the border towards the center of the matrix. These findings were supported by the molecular analyses that revealed a significant increase in the expression of endothelial markers, such as CD31, VEGFA and its receptors, and VWF. Generally, the formation of new capillaries is initiated by growth factors [21]; however, in our model, the HA-based scaffold has fully mimicked the native ECM which in turn allowed the capillaries of surrounding native omentum to integrate and promote the engraftment of the engineered tissue. The vascularization of engineered tissue grafts is often inadequate, thus obstructing the survival of cells which perish due to lack of oxygen and nutrients, as well as insufficient removal of waste products [22]. Moreover, our results demonstrated that the pocket-scaffolds enriched with hMSCs have ensured the metabolic function of the rat hepatocytes, which were able to produce albumin.

Taken together, our results demonstrate that the presence of hMSCs within the pocket-scaffold is fundamental to guarantee its engraftment. Indeed, hMSCs assured a better and efficient modulation of factors involved in neo-angiogenesis, adhesion, migration, proliferation, and differentiation of endothelial cells $[23,24]$. We hypothesize that hMSCs of the pocket-scaffold promoted the neo-angiogenesis by increasing the migration of cells from the surrounding host tissue, and by supporting the proliferation and differentiation of endothelial cells. Promoting the formation of new blood vessels, the hMSCs also supported hepatocytes vitality and function. This was confirmed by a higher metabolic activity of hepatocytes observed only when the pocket-scaffold was enriched with hMSCs. It is proposed that hMSCs can play a key role in releasing trophic factors, which are important in cell protection and support following injury [25]. In our previous in vitro study, we have already demonstrated that hMSCs support hepatocytes maturation in a bioreactor system [26]. In this work, we additionally proved that also the HA-based scaffold plays a critical role in promoting the proliferation of endothelial cells, especially in the area surrounding the scaffold.

\section{Experimental Section}

\subsection{Biomaterial}

In this study, a material derived from the total esterification of HA with benzyl alcohol (HYAFF-11 ${ }^{\mathrm{TM}}$, Fidia Advanced Biopolymers, Abano Terme, Padova, Italy) has been used. The product is a no-crosslinked linear polymer of unknown molecular weight, that is insoluble in aqueous solution but hydrolyzes spontaneously over time, releasing benzyl alcohol and HA. The properties of these substrates are 
described in detail elsewhere [17]. HYAFF- $11^{\mathrm{TM}}$ was used in flexible non-woven meshes (50 $\mu \mathrm{m}$-thick fibers, specific weight of $100 \mathrm{~g} / \mathrm{m}^{2}$ ) creating a final tube-shape.

\subsection{Animals}

Livers of 8 male Lewis rats weighing 200 to $350 \mathrm{~g}$ were used for hepatocyte harvesting. In addition, 12 male athymic nude rats weighing 250 to $400 \mathrm{~g}$ undergone surgery for testing cultured scaffolds. The animals were obtained from Charles River Laboratories (Wilmington, MA, USA); they were maintained on a 12 hours light-dark cycle and given rat chow and water ad libitum. All procedures were approved by the Institutional Animal Care and Use Committee of the University of Padova (Padova, Italy), and performed according to the National Institutes of Health Guidelines for the care and use of laboratory animals.

\subsection{Cells}

\subsection{1. hMSCs isolation from Adipose Tissue}

hMSCs were extracted from adipose tissues of five healthy women and five healthy men (age ranging from 21 to 36 years old; BMI ranging from 30 to 38 ) undergoing cosmetic surgery procedures, according to the guidelines of the plastic surgery clinic at the University of Padova. Written informed consent was obtained from all subjects, in accordance with the Helsinki Declaration. The Ethical Committee of Padova Hospital approved the research protocol. The adipose tissues were digested and the cells isolated and expanded as previously described [27]. Briefly, the adipose tissue was washed with phosphate buffered saline (PBS, EuroClone, Milan, Italy) and digested using a solution of 0.075\% collagenase from Clostridium histolyticum type II (Sigma-Aldrich, St. Louis, MO, USA) in Hank's balanced salt solution (HBSS, Lonza S.r.l., Milano, Italy), for $3 \mathrm{~h}$ at room temperature and under slow agitation. At the end of the digestion, the collagenase activity was blocked with an equal volume of cDMEM, which consisted of Dulbecco's modified Eagle's medium (DMEM, Lonza S.r.1.) supplemented with 10\% fetal bovine serum (FBS, Bidachem S.p.A., Milano, Italy) and 1\% Penicillin/ Streptomycin (P/S, EuroClone). After centrifugation for $4 \mathrm{~min}$ at $1200 \mathrm{rpm}$, the pellet was washed in PBS and filtered with a sterile $70 \mu \mathrm{m}$ cell strainer (BD Biosciences, Mississauga, Ontario, Canada). The cells were transferred to a $25 \mathrm{~cm}^{2}$ tissue culture flask in cDMEM and incubated at $37{ }^{\circ} \mathrm{C}$ and $5 \%$ $\mathrm{CO}_{2}$. After 3 days, floating cells were discarded and fresh medium was added on the adherent cells.

\subsubsection{Hepatocytes isolation from Rat Liver}

Rat hepatocytes were isolated from livers of 8 male Lewis rats, weighing 200 to $350 \mathrm{~g}$. Liver tissue underwent collagenase digestion as previously described by Seglen and coworkers [28]. Briefly, liver tissues were isolated and perfused with $50 \mathrm{~mL}$ of calcium-free PBS (EuroClone), warmed to $37^{\circ} \mathrm{C}$ prior to use. Then, liver tissues were digested in a mix of trypsin (EuroClone) and collagenase (Sigma-Aldrich) at $37^{\circ} \mathrm{C}$. This resulted in blanching, softening, and dissociation of hepatic tissue and provided complete digestion of the liver in $20 \mathrm{~min}$. Hepatocytes were released by mincing and pipetting with a large-bore pipette. The cell suspension was filtered through a sterile $100 \mu \mathrm{m}$ cell strainer (BD Biosciences) into a tube placed on ice, and centrifuged at $1200 \mathrm{rpm}$ for 5 minutes. 
The cells were washed two times in cold PBS and transferred to a $25 \mathrm{~cm}^{2}$ tissue culture flask. The hepatocytes were cultured in DMEM-Ham's F12 (EuroClone) supplemented with 1\% Glutamine (EuroClone), $1 \% \mathrm{P} / \mathrm{S}$, and $10 \% \mathrm{FBS}$.

\subsection{D-cultures preparation}

Three different 3D-cultures were prepared by seeding cells onto HA-based tubes: a) mono-culture of rat hepatocytes; b) mono-culture of hMSCs; c) co-culture of rat hepatocytes and hMSCs (ratio 1:1). Each HA-based tube was seeded with $1 \times 10^{6}$ viable cells, previously mixed with $500 \mu \mathrm{L}$ of fibrin glue (Baxter AG, Vienna, Austria). All 3D-cultures were maintained with DMEM supplemented with 10\% FBS, $1 \%$ glutamine, and $10 \mathrm{nM}$ dexamethasone in a bioreactor at $37{ }^{\circ} \mathrm{C}$ and $5 \% \mathrm{CO}_{2}$. The bioreactor consisted in eight independent chambers, as previously described [26]. The seeded HA-based tubes were placed in the bioreactor, one in each separate chamber, where a perfusion medium flow rate of $0.5 \mathrm{~mL} / \mathrm{min}$ through each scaffold was assured.

All cell-seeded scaffolds were maintained in the bioreactor up to 4 days. At this point, half of the scaffolds were implanted in vivo, whereas the other half was left in the bioreactor for other 7 days.

\subsection{MTT assay}

To determine the proliferation rate of cells grown on HA-based tubes, the MTT (methyl thiazolyl-tetrazolium)-based cytotoxicity assay was performed according to the method of Denizot and Lang with minor modifications [29]. The test is based on mitochondria viability, i.e., only functional mitochondria can oxidize an MTT solution, giving a typical blue-violet end product. After harvesting the culture medium, the cells were incubated for $3 \mathrm{~h}$ at $37{ }^{\circ} \mathrm{C}$ in $1 \mathrm{~mL}$ of $0.5 \mathrm{mg} / \mathrm{mL}$ MTT solution prepared in PBS. After removal of the MTT solution by pipette, $0.5 \mathrm{~mL}$ of $10 \%$ dimethyl sulfoxide in isopropanol was added for $30 \mathrm{~min}$ at $37{ }^{\circ} \mathrm{C}$. For each sample, absorbance values at $570 \mathrm{~nm}$ were recorded in duplicate on $200 \mu \mathrm{L}$ aliquots deposited in 96-well plates using a multilabel plate reader (Victor 3, Perkin Elmer, Milano, Italy). 3D-cultures were examined at 4 and 11 days from the seeding.

\subsection{Albumin ELISA assay}

Albumin secreted by the cells was quantified using the AssayMax Rat Albumin ELISA Kit (Gentaur, Brussels, Belgium). This assay employs a quantitative sandwich enzyme immunoassay technique that measures rat albumin in cell culture medium. A polyclonal antibody specific for rat albumin has been pre-coated onto a 96-well microplate. Albumin in standards and culture media was sandwiched by the immobilized polyclonal antibody and biotinylated polyclonal antibody specific for rat albumin, which was recognized by a streptavidin-peroxidase conjugate. All unbound material was then washed away and a peroxidase enzyme substrate was added. The color development was measured on a microplate reader (Victor 3, Perkin Elmer) at wavelength of $450 \mathrm{~nm}$.

Cell culture media were harvested from the circulating medium in bioreactor at 4 and 11 days, then frozen at $-20{ }^{\circ} \mathrm{C}$ until the analysis. The minimum detectable dose of albumin with this kit is typically $0.7 \mathrm{ng} / \mathrm{mL}$. 


\subsection{D-cultures surgical implantation}

The scaffolds, four for each type of 3D-cultures, were implanted in athymic nude rats up to 7 days. Rats were subjected to surgery under isoflurane anesthesia and allowed to recover after surgical procedure. Implantation was obtained by making small mid-line abdominal incision, and scaffold implantation was performed with externalized omentum which was gently wrapped around the seeded HA-based tube, constituting a final pocket structure. All operations were performed under sterile conditions.

\subsection{H/E and IF stainings}

The pocket-scaffolds were fixed in $4 \%$ phosphate-buffered formalin $\mathrm{pH} 7$, embedded in paraffin, then cut into $7 \mu \mathrm{m}$ thick-sections.

For $\mathrm{H} / \mathrm{E}$ staining, the sections were stained with the nuclear dye hematoxylin (Sigma-Aldrich), and the counterstain eosin (Sigma-Aldrich). For IF staining, the sections were incubated in $2 \%$ bovine serum albumin (BSA, Sigma-Aldrich) solution in PBS for $30 \mathrm{~min}$ at room temperature. The sections were then incubated with the primary antibodies in $2 \%$ BSA solution in a humidified chamber overnight at $4{ }^{\circ} \mathrm{C}$. The following primary antibodies were used: rabbit anti-rat Albumin antibody 1:100 (Bethyl Laboratories, Inc., Montgomery, TX, USA), rabbit anti-Von Willebrand factor antibody 1:100 (Dako, Milan, Italy), and mouse anti-Vimentin antibody 1:100 (Abcam, Cambridge, UK). IF staining was performed with secondary antibodies: anti-rabbit IgG DyLight 488 labeled (KPL, Gaithersburg, MD, USA), and anti-mouse IgG DyLight 549 labeled (KPL) in 2\% BSA for $1 \mathrm{~h}$ at room temperature. Nuclear staining was performed with $2 \mu \mathrm{g} / \mathrm{mL}$ Hoechst H33342 (Sigma-Aldrich) solution for $5 \mathrm{~min}$. The sections were coverslipped with a drop of mounting medium.

\subsection{Real-time PCR}

Total RNA was extracted from each sample by using the TRIzol ${ }^{\circledR}$ Reagent (Invitrogen, Carlsbad, CA, USA). The samples were quantified using the NanoDrop spectrophotometer (NanoDrop ${ }^{\text {TM }} 1000$, Thermo Scientific, Waltham, MA, USA). For the first-strand cDNA synthesis, $500 \mathrm{ng}$ of total RNA was reverse transcribed using M-MLV RT (Moloney Murine Leukemia Virus Reverse Transcriptase, Invitrogen, Paisley, UK) according to the manufacturer's protocol. Primers were selected for each target gene with Primer 3 software. Real-time PCRs were carried out using the designed primers at a concentration of $300 \mathrm{nM}$ and FastStart SYBR Green Master (Roche Diagnostics, Mannheim, Germany) on a Rotor-Gene 3000 (Corbett Research, Sydney, Australia). Thermal cycling conditions were as follows: 15 min denaturation at $95{ }^{\circ} \mathrm{C}$; followed by 40 cycles of $15 \mathrm{~s}$ denaturation at $95{ }^{\circ} \mathrm{C}$; annealing for $30 \mathrm{~s}$ at $60{ }^{\circ} \mathrm{C}$; and $20 \mathrm{~s}$ elongation at $72{ }^{\circ} \mathrm{C}$. Values were normalized to the expression of the $\beta$-actin internal reference, whose abundance did not change under our experimental conditions. Experiments were performed with 3 different cell preparations and repeated at least 3 times.

\subsection{Statistical analysis}

One-way analysis of variance (ANOVA) was used to analyze the data. A post-hoc analysis using Bonferroni's multiple comparison test was used to identify any significant changes $(* \mathrm{p}<0,05)$. 
Repeatability was calculated as the standard deviation of the difference between measurements. All tests were performed using the SPSS 16.0 software package (SPSS Inc., Chicago, IL, USA) (licensed to the University of Padova, Italy).

\section{Conclusions}

The clinical success of an engineered tissue graft is very limited without a proper vascularization. To overcome this problem, the stimulation of angiogenesis is the main strategy to ensure the vascularization of engineered grafts. The formation of new blood vessels is facilitated by biomaterials with 3D structure that mimics the native ECM. For this reason, the development of scaffolds equivalent to native ECM represents an important investigation area in the field of vascularized tissue engineering. However, the full restoration of standard vascular function and structural integrity represents a complex aim to achieve because of native ECM complexity and the lack of spatial and temporal integrity of the grafts [30].

In the present work, we have demonstrated that a HA-based scaffold can promote the vascularization of tissue engineered grafts, especially in the border areas. This property must be added to the already known characteristic of low antigenicity, biodegradability, and good mechanical and hemostatic properties [17]. Our results also demonstrate the important role played by hMSCs in neoangiogenesis and metabolic process. Indeed, hMSCs increased growth factors in the pocket-scaffold, promoted angiogenesis resulting in a high blood vessel density coupled with a better support to metabolic function of hepatocytes.

In conclusion, a scaffold with excellent properties enriched with hMSCs could be considered a smart approach to increase the engrafting of engineered tissues. This modification may induce a guided formation of blood vessels through the migration and proliferation of endothelial cells within the graft. In turn, the neo-angiogenesis may guarantee oxygen and nutrients, as well as the removal of waste products within the graft. Thus, hMSCs seem to support vitality and metabolic functions of hepatocytes creating a proper environment.

\section{Author Contributions}

Conceived and designed the experiments: Barbara Zavan, Umberto Tedeschi, and Amedeo Carraro; Performed the experiments: Letizia Ferroni, Chiara Gardin, and Maurizio Buggio; Performed the surgical procedures: Amedeo Carraro, and Maurizio Buggio; Analyzed the data: Letizia Ferroni, Chiara Gardin, and Barbara Zavan; Contributed reagents/materials/analysis tools: Barbara Zavan; Wrote the paper: Amedeo Carraro, Chiara Gardin, Letizia Ferroni, and Barbara Zavan.

\section{Conflicts of Interest}

The authors declare no conflict of interest.

\section{References and Notes}

1. Lee, W.M.; Squires, R.H. Jr.; Nyberg, S.L.; Doo, E.; Hoofnagle, J.H. Acute liver failure: Summary of a workshop. Hepatology 2008, 47, 1401-1415. 
2. Yu, C.B.; Pan, X.P.; Li, L.J. Progress in bioreactors of bioartificial livers. Hepatobiliary Pancreat. Dis. Int. 2009, 8, 134-140.

3. Van de Kerkhove, M.P.; Poyck, P.P.; Deurholt, T.; Hoekstra, R.; Chamuleau, R.A.; van Gulik, T.M. Liver support therapy: an overview of the AMC-bioartificial liver research. Dig. Surg. 2005, 22, 254-264.

4. Morsiani, E.; Brogli, M.; Galavotti, D.; Pazzi, P.; Puviani, A.C.; Azzena, G.F. Biologic liver support: optimal cell source and mass. Int. J. Artif. Organs 2002, 25, 985-993.

5. Van de Kerkhove, M.P.; Hoekstra, R.; Chamuleau, R.A.; van Gulik, T.M. Clinical application of bioartificial liver support systems. Ann. Surg. 2004, 240, 216-230.

6. Inamori, M.; Mizumoto, H.; Kajiwara, T. An approach for formation of vascularized liver tissue by endothelial cell-covered hepatocyte spheroid integration. Tissue Eng. Part A 2009, 15, 2029-2037.

7. Tilles, A.W.; Berthiaume, F.; Yarmush, M.L.; Toner, M. Critical issues in bioartificial liver development. Technol. Health Care 2002, 10,177-186.

8. Discher, D.E.; Mooney, D.J.; Zandstra, P.W. Growth factors, matrices, and forces combine and control stem cells. Science 2009, 324, 1673-1677.

9. Spradling, A.; Drummond-Barbosa, D.; Kai, T. Stem cells find their niche. Nature 2001, 414, 98-104.

10. Korff, T.; Augustin, H.G. Integration of endothelial cells in multicellular spheroids prevents apoptosis and induces differentiation. J. Cell Biol. 1998, 143, 1341-1352.

11. Montaño, I.; Schiestl, C.; Schneider, J.; Pontiggia, L.; Luginbühl, J.; Biedermann, T.; Böttcher-Haberzeth, S.; Braziulis, E.; Meuli, M.; Reichmann, E. Formation of human capillaries in vitro: the engineering of prevascularized matrices. Tissue Eng. Part A. 2010, 16, 269-282.

12. Bartneck, M.; Topuz, F.; Tag, C.G.; Sauer-Lehnen, S.; Warzecha, K.T.; Trautwein, C.; Weiskirchen, R.; Tacke, F. Molecular response of liver sinusoidal endothelial cells on hydrogels. Mater. Sci. Eng. C Mater. Biol. Appl. 2015, 51, 64-72.

13. Luong, E.; Gerecht, S. Stem cells and scaffolds for vascularizing engineered tissue constructs. Adv. Biochem. Eng. Biotechnol. 2009, 114,129-172.

14. Bressan, E.; Carraro, A.; Ferroni, L.; Gardin, C.; Sbricoli, L.; Guazzo, R.; Stellini, E.; Roman, M.; Pinton, P.; Sivolella, S.; Zavan, B. Nanotechnology to drive stem cell commitment. Nanomedicine (Lond) 2013 , 8, 469-486.

15. McMurray, R.J.; Gadegaard, N.; Tsimbouri, P.M.; Burgess, K.V.; McNamara, L.E.; Tare, R.; Murawski, K.; Kingham, E.; Oreffo, R.O.; Dalby, M.J. Nanoscale surfaces for the long-term maintenance of mesenchymal stem cell phenotype and multipotency. Nat Mater. 2011, 10, 637-644.

16. Reilly, G.C.; Engler, A.J. Intrinsic extracellular matrix properties regulate stem cell differentiation. J. Biomech. 2010, 43, 55-62.

17. Vindigni, V.; Cortivo, R.; Iacobellis, L.; Abatangelo, G.; Zavan, B. Hyaluronan benzyl ester as a scaffold for tissue engineering. Int. J. Mol. Sci. 2009, 10, 2972-2985.

18. Gardin, C.; Vindigni, V.; Bressan, E.; Ferroni, L.; Nalesso, E.; Puppa, A.D.; D'Avella D.; Lops, D.; Pinton, P.; Zavan, B. Hyaluronan and fibrin biomaterial as scaffolds for neuronal differentiation of adult stem cells derived from adipose tissue and skin. Int. J. Mol. Sci. 2011, 12, 6749-6764. 
19. Garcia-Fuentes, M.; Meinel, A.J.; Hilbe, M.; Meinel, L.; Merkle, H.P. Silk fibroin/hyaluronan scaffolds for human mesenchymal stem cell culture in tissue engineering. Biomaterials 2009, 30, 5068-5076.

20. Girotto, D.; Urbani, S.; Brun, P.; Renier, D.; Barbucci, R.; Abatangelo, G. Tissue-specific gene expression in chondrocytes grown on three-dimensional hyaluronic acid scaffolds. Biomaterials 2003, 24, 3265-3275.

21. Herrmann, M.; Verrier, S.; Alini, M. Strategies to Stimulate Mobilization and Homing of Endogenous Stem and Progenitor Cells for Bone Tissue Repair. Front. Bioeng. Biotechnol. 2015, 3, 79.

22. Baiguera, S., Ribatti, D. Endothelialization approaches for viable engineered tissue. Angiogenesis 2013, 16, 1-14.

23. Kaully, T.; Kaufman-Francis, K.; Lesman, A.; Levenberg, S. Vascularization-the conduit to viable engineered tissues. Tissue Eng. Part B Rev. 2009, 5, 159-169.

24. Lokmic, Z.; Mitchell, G.M. Engineering the microcirculation. Tissue Eng. Part B Rev.2008, 14, 87-103.

25. Yu, B.; Zhang, X.; Li, X. Exosomes derived from mesenchymal stem cells. Int. J. Mol. Sci. 2014, $15,4142-4157$.

26. Carraro, A.; Flaibani, M.; Cillo, U.; Michelotto, L.; Magrofuoco, E.; Buggio, M.; Abatangelo, G.; Cortivo, R.; Herrera, M.B.; Tetta, C.; Elvassore, N.; Zavan, B. A combining method to enhance the in vitro differentiation of hepatic precursor cells. Tissue Eng. Part C Methods. 2010, 16, $1543-1551$.

27. Clauser, L.; Ferroni, L.; Gardin, C.; Tieghi, R.; Galiè, M.; Elia, G.; Piattelli, A.; Pinton, P.; Bressan, E.; Zavan, B. Selective augmentation of stem cell populations in structural fat grafts for maxillofacial surgery. PLoS One 2014, 9, e110796.

28. Seglen, P.O. Preparation of isolated rat liver cells. Methods Cell Biol. 1976, 13, 29-83.

29. Rigo, C.; Ferroni, L.; Tocco, I.; Roman, M.; Munivrana, I.; Gardin, C.; Cairns, W.R.; Vindigni, V.; Azzena, B.; Barbante, C.; Zavan, B. Active silver nanoparticles for wound healing. Int. J. Mol. Sci. 2013, 14, 4817-4840.

30. Novosel, E.C.; Kleinhans, C.; Kluger, P.J. Vascularization is the key challenge in tissue engineering. Adv. Drug Deliv. Rev. 2011, 63, 300-311.

(C) 2015 by the authors; licensee MDPI, Basel, Switzerland. This article is an open access article distributed under the terms and conditions of the Creative Commons Attribution license (http://creativecommons.org/licenses/by/4.0/). 